\title{
The Sampling Theorem for Functions with Limited Multi-Band Spectrum I
}

\author{
L. Bezuglaya and V. KATSNELSON
}

In this paper functions $f$ belonging to $L^{2}(\mathbb{R})$ are considered which spectrum is contained in a 'multi-band' set $E$, i.e. in a subset of the real axis which is the union of finite many intervals. For such functions a generalization of the Whittaker-Kotelnikov-Shannon sampling formula is given. The considered problem is also related to Riesz bases of exponentials in $L^{2}(E)$. In the first part of this work we consider sets $E$ consisting of regularly positioned intervals of the same length.

Key words: . Multi-band spectrum, square-summable entire functions, interpolation of entire functions, the sampling formula, bases of exponentials in $L^{2}$, cardinal series

AMS subject classification: 30D10, 30D15, 46B15, 94A12

\section{Introduction. The Whittaker-Kotelnikov-Shannon sampling theorem}

The following result is well known (see, for example, [8, item 2.9], [11, Chapter 2], [19, Chapter 3]):

Let $f$ be a function which belongs to $L^{2}(\mathbb{R})$, i.e. $\int_{\mathbb{R}}|f(x)|^{2} d x<\infty$, and let $f$ be an entire function of exponential type not exceeding $\sigma$ :

$$
\varlimsup_{|z| \rightarrow \infty} \frac{\ln |f(z)|}{|z|} \leq \sigma
$$

where $\sigma$ is a given positive number.

Then the function $f$ is uniquely determined by its values $f\left(\frac{k \pi}{\sigma}\right)$ at the sequence

L. Bezuglaya: The Kharkov State Univ., Dep. Math., Kharkov 310077, Ukraine

V.E. Katsnolson: The Weizmann Institute Sci., Dop. Thoor. Math., Rehovot 76100, Israel 
$\left\{\frac{k \pi}{\sigma}\right\}_{k \in \mathbb{Z}}$ of points of the real axis. The sequence $\left\{f\left(\frac{k \pi}{\sigma}\right)\right\}$ belongs to $\ell^{2}$. Moreover, the Parseval identity holds:

$$
\frac{\pi}{\sigma} \sum_{k \in \mathbb{Z}}\left|f\left(\frac{k \pi}{\sigma}\right)\right|^{2}=\int_{\mathbb{R}}|f(x)|^{2} d x
$$

The function $f$ can be reconstructed from the sequence $\left\{f\left(\frac{k \pi}{\sigma}\right)\right\}_{k \in Z}$ of its values by the interpolation series

$$
f(z)=\sum_{k \in \mathbb{Z}} f\left(\frac{k \pi}{\sigma}\right) \frac{\sin \sigma\left(z-\frac{k \pi}{\sigma}\right)}{\sigma\left(z-\frac{k \pi}{\sigma}\right)} .
$$

The series converges both in $L^{2}(\mathbb{R})$ and locally uniformly in the complex plane. The interpolation (1.9) is 'free': The sequence $\left\{f\left(\frac{k \pi}{\sigma}\right)\right\}_{k \in \mathbb{Z}}$ can be an arbitrary sequence $C=\left\{c_{k}\right\}_{k \in \mathbb{Z}} \in \ell^{2}$, that is $\sum_{k \in \mathbb{Z}}\left|c_{k}\right|^{2}<\infty$. For each sequence $\left\{c_{k}\right\}_{k \in \mathbb{Z}} \in \ell^{2}$ the series

$$
f_{c}(z)=\sum_{k \in \mathbb{Z}} c_{k} \frac{\sin \sigma\left(z-\frac{k \pi}{\sigma}\right)}{\sigma\left(z-\frac{k \pi}{\sigma}\right)}
$$

converges both locally uniformly and in $L^{2}(\mathbb{R})$ and defines an entire function $f_{c}$ of an exponential type not exceeding $\sigma$ which satisfies the interpolation conditions $f_{c}\left(\frac{k \pi}{\sigma}\right)=$ $c_{k}(k \in \mathbb{Z})$. Moreover, the Parseval identity

$$
\int_{\mathbb{R}}\left|f_{c}(x)\right|^{2} d x=\frac{\pi}{\sigma} \sum_{k \in \mathbb{Z}}\left|c_{k}\right|^{2}
$$

holds.

Thus, every sequence from $\ell^{2}$ can appear as the sequence $\left\{f\left(\frac{k \pi}{\sigma}\right)\right\}$ of the values of an entire function $f \in L^{2}(\mathbb{R})$ satisfying the condition (1.1):

Each function $f \in L^{2}(\mathbb{R}, d x)$ is representable in the form

$$
f(x)=\int_{\mathbb{R}} \varphi(\lambda) e^{i \lambda x} d \lambda
$$

The Fourier-Plancherel transform $\varphi$ of $f$ also belongs to $L^{2}(\mathbb{R}, d \lambda)$, and the equality $\int_{\mathbb{R}}|\varphi(\lambda)|^{2} d \lambda=\frac{1}{2 \pi} \int_{\mathbb{R}}|f(x)|^{2} d x$ holds. The integral over $\mathbb{R}$ in (1.5) is defined, as an improper one, namely as the square mean limit of the integral over $(-N, N)$ as $N \rightarrow \infty$. In its turn, the function $\varphi$ is the inverse Fourier transform of the function $f$, i.e. $\varphi(\lambda)=\frac{1}{2 \pi} \int_{\mathbb{R}} f(x) e^{-i \lambda x} d x$ (in general, this integral is also improper: 1.i.m.). 
The Wiener-Paley theorem states that a function $f \in L^{2}(\mathbb{R})$ is the restriction on the real axis of an entire function $f$ of exponential type not exceeding $\sigma$ (i.e. $f$ satisfies the condition (1.1)) if and only if the condition

$$
\varphi(\lambda)=0 \quad \text { for all } \lambda \bar{\epsilon}[-\sigma, \sigma]
$$

holds. This condition can be formulated in terms of the notion 'spectrum of the function'. Let $\varphi$ be a measurable function. By definition, a real point $\lambda_{0}$ belongs to the support of the function $\varphi$ if, for any $\varepsilon>0$, the set $\left(\lambda_{0}-\varepsilon, \lambda_{0}+\varepsilon\right) \cap\{\lambda: \varphi(\lambda) \neq 0\}$ has positive Lebesgue measure. Thus, the condition (1.6) can be expressed in such a way: the support of the function $\varphi$ is contained in the interval $[\sigma, \sigma]$.

Definition: The spectrum of a function $f$ belonging to $L^{2}(\mathbb{R})$ is the support of its Fourier transform $\varphi$.

If we interpret the variable $x$ as time it is natural to consider the number $\lambda$ in the exponent $e^{i \lambda x}$ as frequency. In this connection it is natural to call the spectrum of the function $f$ its frequency spectrum.

The assertion stated above can be formulated also in spectral language:

Let $f \in L^{2}(\mathbb{R})$ be a function with spectrum contained in the interval $[-\sigma, \sigma]$. . Then $f$ is uniquely determined by its values $f\left(\frac{k \pi}{\sigma}\right)$ at the points of the sequence $\left\{\frac{k \pi}{\sigma}\right\}_{k \in \mathbb{Z}}$, and $f$ can be recovered from these values by means of the interpolation series (1.9). The sequence $\left\{f\left(\frac{k \pi}{\sigma}\right)\right\}_{k \in \mathbb{Z}}$ belongs to $\ell^{2}$, and the Parseval identity (1.2) holds. An arbitrary sequence from $\ell^{2}$ can appear as the sequence $\left\{f\left(\frac{k \pi}{\sigma}\right)\right\}_{k \in \mathbb{Z}}$.

Expansions of entire functions via Lagrange interpolation series (1.3) were considered by E.T. Whittaker long ago, as long ago as in 1915 (see [30]). (See also J.M. Whittaker's book [31].) What concerns early publication on this subject, the paper [12] by G.H. Hardy should be mentioned. In 1933 V.A. Kotelnikov paid attention to the fundamental significance of the expansion (1.3) for the transmission information theory. He has formulated the following fundamental proposition:

Let a signal be described by a function $f$ with a bounded frequency spectrum which is contained in $[-\sigma, \sigma]$ (that is, this signal contains no frequencies higher than $\frac{\sigma}{2 \pi}$ cycles per second). Let us have a certain communication channel. For the possibility of recovering this signal at the output of this communication channel it is sufficient to transmit over this channel only the values $f(k \Delta)$ of this signal at the sampling points $\Delta$ apart, if $\Delta=\frac{\pi}{\sigma}$.

The formula (1.3) and the above statement present the content of two fundamental 
theorems of V.A. Kotelnikov which are contained in his work [20]. In the Russian scientific literature these statements are combined and called 'Theorem of Kotelnikov'. In the western scientific literature these statements are called 'The Sampling Theorem'. C. Shannon has used the sampling theorem in his work [28] in the communication theory. H. Nyquist [26] has pointed out the significance of the interval $\Delta=\frac{\pi}{\sigma}$ for the telegraphy. C. Shannon called this interval Nyquist interval corresponding to the frequency band $[-\sigma, \sigma]$.

Of course, it is possible also to use the values $\left\{f\left(\gamma+k \Delta_{1}\right\}_{k \in \mathbb{Z}}\right.$ at an arbitrary periodic sampling sequence with an arbitrary $\Delta_{1}: 0<\Delta_{1}<\Delta=\frac{\pi}{\sigma}$. This is true since if the spectrum of a function is contained in the interval $[-\sigma, \sigma]$, then, all the more, this spectrum is contained in the interval $\left[-\sigma_{1}, \sigma_{1}\right]$ where $\sigma_{1}>\sigma$.

If $x_{k}=\gamma+k \frac{\pi}{\sigma_{1}}\left(\gamma\right.$ a real number, $\left.\sigma_{1}>\sigma\right)$, and $f \in L^{2}(\mathbb{R})$ is a function which spectrum is contained in $[-\sigma, \sigma]$, then the equality

$$
f(x)=\sum_{k \in \mathbb{Z}} f\left(x_{k}\right) \frac{\sin \sigma_{1}\left(x-x_{k}\right)}{\sigma_{1}\left(x-x_{k}\right)}
$$

holds for every complex $x$.

In the Whittaker-Kotelnikov-Shannon Theorem the samplings form an equidistant sequence of real points. However, it is possible to generalize this result and to get analogous results for some sampling sequences which are not equidistant ones. Of course, in such generalizations the equality (1.2) does not remain true and has to be replaced by an inequality of the form

$$
m^{2} \sum_{k \in \mathbb{Z}}\left|f\left(x_{k}\right)\right|^{2} \leq \int_{\mathbb{R}}|f(x)|^{2} d x \leq M \sum_{k \in \mathbb{Z}}\left|f\left(x_{k}\right)\right|^{2}
$$

(For such generalizations, we refer to $[16,18,24,32]$.

Note, that if $\sigma_{1}<\sigma$ (i.e., if $\left[-\sigma_{1}, \sigma_{1}\right] \subset[-\sigma, \sigma]$ ), it is natural to consider the sequence $\left\{\frac{k \pi}{\sigma_{1}}\right\}_{k \in \mathbb{Z}}$ as thinner than the sequence $\left\{\frac{k \pi}{\sigma}\right\}_{k \in \mathbb{Z}}$. In other words, if it is known that the spectrum of a function $f$ is contained not only in the interval $[-\sigma, \sigma]$ but in fact in a more narrow interval $\left[-\sigma_{1}, \sigma_{1}\right]$, then it is possible to use a thinner sequence of samplings for recovering this function. Detailed surveys of results concerning the sampling theorem and its various extensions and applications are presented in $[4,5,13$, $17,29]$. 


\section{The sampling theorem for functions with multi-band spec- trum}

The general statement of the question under consideration is as follows. Let $E$ be a closed subset of the real axis, with positive Lebesgue measure. Let $f \in L^{2}(\mathbb{R}, d x)$ be a function whose spectrum is contained in the set $E$. Which condition must a discrete sequence $\left\{x_{k}\right\} \subset \mathbb{R}$ satisfy in order to ensure that $f$ can be recovered from the sequence $\left\{f\left(x_{k}\right)\right\}$ of its values? How is it possible to realize such a recovery? It is desirable to get the solution of this problem in the form of an interpolation series. It is also desirable that such an interpolation' would be 'free', i.e., that it would be possible to choose an arbitrary sequence (from $\ell^{2}$ ) as the sequence $\left\{f\left(x_{k}\right)\right\}$.

Of course, if $E$ is a subset of the interval $[-\sigma, \sigma]$, then it is possible to choose $\left\{x_{k}\right\}=\left\{\frac{k \pi}{\sigma}\right\}$ as such a sequence. (The sequence $\left\{x_{k}\right\}=\left\{\frac{k \pi}{\sigma}\right\}$ corresponds to the whole interval $[-\sigma, \sigma]$.) However, by such a choice of the sequence $\left\{x_{k}\right\}$ the interpolation is not 'free'. If the spectrum of $f$ belongs to a proper closed subset $E$ of the interval $[-\sigma, \sigma]$, the sequence $\left\{f\left(\frac{k \pi}{\sigma}\right)\right\}_{k \in \mathbb{Z}}$ cannot be an arbitrary square summable one. As the set $E$ is smaller than the whole interval $[-\sigma, \sigma]$, it is natural to try to find sequences $\left\{x_{k}\right\}$ which are 'less dense' than the sequence $\left\{\frac{k \pi}{\sigma}\right\}$. (It is desirable to find sequences which are simultaneously sufficiently dense for the possibility of the recovery of the function $f$ from its values $\left\{f\left(x_{k}\right)\right\}$ and which are at the same time sufficiently thin for the interpolation to be free.)

In this article a special case of this problem is considered, namely we will specify the form of the set $E$. The set $E$ will be assumed as a finite union of intervals. These intervals are positioned regularly and are supposed to have the same length.

Let $p$ and $q$ be integers satisfying $0<p<q$. We separate the interval $[-\sigma, \sigma]$ into $q$ equal intervals. (Each of these intervals has the length $\frac{2 \sigma}{q}$.) The set. $E$ is the union of $p$ of these $q$ intervals. Thus, the Lebesgue measure mes $E$ of the set $E$ is mes $E=2 \sigma \underset{q}{p}$. (The intervals forming the set $E$ can have common end points and form after confluence bigger intervals which do not intersect and which length is a multiple of $\frac{2 \sigma}{q}$.)

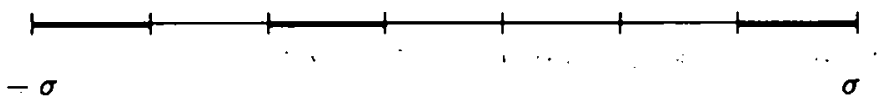

Figure 1.

Figure 1 corresponds to $q=7, p=3$, in which case the intervals form a set $E$ without common end points.

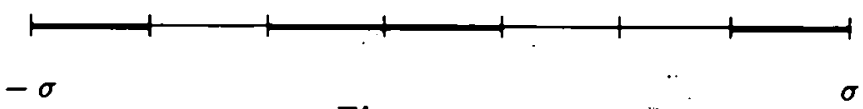

Figure 2. 
Figure 2 corresponds to $q=7, p=4$, in which case two of the four intervals have common end points.

Thus the spectrum of the considered functions consists of many 'bands', namely of those intervals which form the set $E$. (This is the motivation for the title of this paper.)

Here we get an interpolation formula (the so-called sampling formula) for a function $f \in L^{2}(\mathbb{R}, d x)$, which spectrum is contained in such a multi-band set $E$. The sequence $\left\{x_{k}\right\}$ of samplings will be $\frac{p}{q}$ times thinner than the sequence $\left\{\frac{k \pi}{\sigma}\right\}$ which corresponds to the whole interval $[\sigma, \sigma]$. In detail, the sequence consists of groups, each of which consists of $p$ real points, and these groups are positioned periodically, with period $q \frac{2 \pi}{\sigma}$.

Let $\lambda_{n}(n=1,2, \ldots, p)$ be the centers of the intervals $I_{n}$ consisting of the set $E, \lambda_{n^{\prime}} \neq \lambda_{n^{\prime \prime}}$ for $n^{\prime} \neq n^{\prime \prime}$. We suppose the points $\lambda_{n}$ to be distributed 'regularly', that is

$$
\bigcup_{1 \leq n \leq p}\left\{\lambda_{n}\right\} \subseteq \bigcup_{0 \leq \ell \leq q-1}\left\{-\sigma+(2 \ell+1) \frac{\sigma}{q}\right\}
$$

Thus,

$$
I_{n}=\left[\dot{\lambda_{n}}-\frac{\sigma}{q}, \lambda_{n}+\frac{\sigma}{q}\right], \text { and } E=\bigcup_{1 \leq n \leq p} I_{n}
$$

Before deriving the sampling formula for a function with spectrum in $E$ we recall how to get the 'usual' sampling formula for a function with spectrum in $[-\sigma, \sigma]$. So, let the spectrum of $f$ be contained in $[-\sigma, \sigma]$, that is

$$
f(x)=\int_{[-\sigma, \sigma]} \varphi(\lambda) e^{i \lambda x} d \lambda
$$

where $\varphi \in L^{2}[\sigma, \sigma]$. We expand the exponential function in a Fourier series with respect to the system $\left\{e^{i k \frac{\pi}{\sigma} \lambda}\right\}_{k \in \mathbb{Z}}$ (this system is an orthogonal basis in $L^{2}(-\sigma, \sigma)$ ):

$$
e^{i \lambda x}=\sum_{k \in \mathbb{Z}} c_{k}(x) e^{i \frac{k \pi}{\sigma} \lambda}, \quad \lambda \in[-\sigma, \sigma] .
$$

The Fourier coefficients $c_{k}(x)$ can be found in a standard way:

$$
2 \sigma c_{k}(x)=\int_{[-\sigma, \sigma]} e^{i \lambda x} e^{-i \frac{k \pi}{\sigma} \lambda} d \lambda, \text { that is } c_{k}(x)=\frac{\sin \sigma\left(x-\frac{k \pi}{\sigma}\right)}{\sigma\left(x-\frac{k \pi}{\sigma}\right)}
$$

Thus,

$$
e^{i \lambda x}=\sum_{k \in \mathbb{Z}} \frac{\sin \sigma\left(x-\frac{k \pi}{\sigma}\right)}{\sigma\left(x-\frac{k \pi}{\sigma}\right)} e^{i \frac{k \pi}{\sigma} \lambda}, \quad \lambda \in[-\sigma, \sigma]
$$


Inserting (2.4) into (2.3) and changing the order of summation and integration we get

$$
f(x)=\sum_{k \in \mathbb{Z}} \frac{\sin \sigma\left(x-\frac{k \pi}{\sigma}\right)}{\sigma\left(x-\frac{k \pi}{\sigma}\right)} \int_{[-\sigma, \sigma]} \varphi(\lambda) e^{i \frac{k \pi}{\sigma} \lambda} d \lambda .
$$

Because of $\int_{[-\sigma, \sigma]} \varphi(\lambda) e^{i \frac{k \pi}{\sigma} \lambda} d \lambda=f\left(\frac{k \pi}{\sigma}\right)$ (this is the formula (2.3) for $x=\frac{k \pi}{\sigma}$ ), we get the formula (1.3).

Note that this proof of the sampling formula is rather different from the generally known versions. Usually one does not uses the Fourier expansion for the exponential function $e^{i \lambda \dot{x}}$ but for the function $\varphi$. (See, for example, [5, item 2.9], [8, §2.1], [15, §3.1].)

Now we start to derive the sampling formula for functions with 'multiband' spectrum. So, let $E$ be a set of the shape (2.1) - (2.2). We want to find a system of exponents $\left\{e^{i \lambda x_{m}}\right\}$ which is an orthogonal basis in the space $L^{2}(E)$. Further, we will expand the exponential function $e^{i \lambda x}$ into a Fourier series on $E$ :

$$
e^{i \lambda x}=\sum_{m \in \mathbb{Z}} c_{m}(x) e^{i \lambda x_{m}} \quad(\lambda \in E)
$$

The following consideration repeats the one used above for obtaining the sampling formula for functions with one-band spectrum. (If we are able to compute the Fourier coefficients $c_{m}$ in (2.5).)

Let $h_{j}(1 \leq j \leq p)$ be (unknown at present) $\frac{2 \sigma}{q}$-periodic functions defined on the real axis: $h_{j}\left(\lambda+\frac{2 \sigma}{q} m\right) \equiv h_{j}(\lambda)(m \in \mathbb{Z}, \lambda \in \mathbb{R}, j=1,2, \ldots, p)$. Let $\gamma_{1}, \gamma_{2}, \ldots, \gamma_{n}$ be real numbers for which it is only assumed that $D \neq 0$ where

$$
D=\operatorname{det}\left\|e^{i \gamma_{j} \lambda_{n}}\right\| \quad(1 \leq j \leq p, \quad 1 \leq n \leq p)
$$

Without loss of generality we can suppose that $\gamma_{1}<\gamma_{2}<\ldots<\gamma_{n}$. We determine the functions $h_{j}$ from the condition $\sum_{1 \leq j \leq p} h_{j}(\lambda) e^{i \gamma_{j} \lambda}=e^{i \lambda x}(\lambda \in E)$, that is we solve the system

$$
\sum_{1 \leq j \leq n} h_{j}(\lambda) e^{i \gamma_{j} \lambda}=e^{i \lambda x} \quad\left(\lambda \in I_{n}, n=1,2, \ldots, p\right)
$$

We suppose that $q$ is odd. In (2.6) there figure up the values of the functions $h_{j}$ on different intervals. Using periodicity of these functions, we can reduce system (2.6) to the system in which there figure up the values $h_{j}$ on one and the same 'supporting' interval. If $q$ is odd, we choose as such supporting interval $I_{0}=\left[-\frac{\sigma}{q}, \frac{\sigma}{q}\right]$. As the functions $h_{j}$ are $\frac{2 \sigma}{q}$-periodic they are completely determined by their restrictions on 
any interval with length $\frac{2 \sigma}{q}$, in particular, on the interval $I_{0}$. In view of $(2.2)$, the points $\lambda$ of the interval $I_{n}$ can be parametrized in the form $\lambda=\lambda_{n}+\mu, \mu \in I_{0}$. As $\lambda_{n}$ is a multiple of $\frac{2 \sigma}{q}$ (see (2.1). $q$ is odd). then $h_{j}\left(\lambda_{n}+\mu\right) \equiv h_{j}(\mu)(1 \leq j \leq p)$. Substituting $\lambda=\lambda_{n}+\mu$ into (2.6), we get the identities

$$
\sum_{1 \leq j \leq p} h_{j}(\mu) e^{i \gamma_{j} \mu} e^{i \gamma_{j} \lambda_{n}}=e^{i \lambda_{n} x} e^{i \mu x} \quad\left(\mu \in I_{0}, 1 \leq n \leq p\right)
$$

We consider these equalities as a system of linear algebraic equations concerning the 'unknown' variables $h_{j}(\mu) e^{i \gamma_{j} \mu}$ with the coefficient matrics $\left\|e^{i \gamma_{j} \lambda_{n}}\right\|$. By assumption, the determinant $D$ of this matrix does not vanish. Solving the system (2.7), we get

$$
h_{n}(\mu) e^{i \gamma_{n} \mu}=\frac{D_{n}(x)}{D} e^{i \mu x}
$$

where $D:=\operatorname{det}\left\|e^{i \lambda_{n} \gamma_{j}}\right\|_{\substack{1 \leq n \leq p \\ 1 \leq j \leq p}}$ and

$$
D_{n}(x)=\operatorname{det}\left[\begin{array}{ccccccc}
e^{i \lambda_{1} \gamma_{1}} & \cdots & e^{i \lambda_{1} \gamma_{n-1}} & e^{i \lambda_{1} x} & e^{i \lambda_{1} \gamma_{n}+1} & \ldots & e^{i \lambda_{1} \gamma_{p}} \\
e^{i \lambda_{2} \gamma_{1}} & \cdots & e^{i \lambda_{2} \gamma_{n-1}} & e^{i \lambda_{2} x} & e^{i \lambda_{2} x_{n}+1} & \cdots & e^{i \lambda_{2} \gamma_{p}} \\
\cdot & \cdot & \cdot & \cdot & \cdot & \cdot & \cdot \\
e^{i \lambda_{p} \gamma_{1}} & \cdots & e^{i \lambda_{p} \gamma_{n}-1} & e^{i \lambda_{p} x} & e^{i \lambda_{p} \gamma_{n+1}} & \cdots & e^{i \lambda_{p} \gamma_{p}}
\end{array}\right]
$$

Evidently, $D_{n}\left(\gamma_{n}\right)=D$ and $D_{n}\left(\gamma_{m}\right)=0 \quad(1 \leq m \leq p, m \neq n)$. (In the corresponding matrix the $m$-th and the $n$-th columns coincide.) From (2.1) it follows that $\exp \left\{i \lambda_{n} \gamma_{j}\right\}=\exp \left\{i \lambda_{n}\left(\gamma_{j}+q \frac{k \pi}{\sigma}\right)\right\}$ for $n=1,2, \ldots, p, j=1,2, \ldots, p$ and for every integer $k$ (we recall that $q$ is odd now). Thus, for odd $q$ the equalities

$$
\begin{gathered}
D_{n}\left(\gamma_{n}+k q \frac{\pi}{\sigma}\right)=D \quad(1 \leq n \leq p, k \text { integer }) \\
D_{n}\left(\gamma_{m}+k q \frac{\pi}{\sigma}\right)=0 \quad(1 \leq m \leq p, \quad m \neq n, k \text { integer })
\end{gathered}
$$

hold. From (2.8) we have

$$
h_{n}(\mu)=\frac{D_{n}(x)}{D} e^{i \mu\left(x-\gamma_{n}\right)} \quad\left(n=1, \ldots, p ; \mu \in I_{0}\right) .
$$

Expanding the function $h_{n}=h_{n}(\mu)$ into a Fourier series with respect to the system $\left\{\exp \left(i \kappa q \frac{\pi}{\sigma} \mu\right)\right\}_{k \in \mathbb{Z}}$, which is an orthogonal basis in $L^{2}\left(I_{0}\right)$, and computing the Fourier coefficients in standard way, we get the equalities

$$
h_{j}(\mu)=\frac{D_{j}(x)}{D} \sum_{k \in \mathbb{Z}} \frac{\sin \frac{\sigma}{q}\left(x-\gamma_{j}-q \frac{\pi}{\sigma} k\right)}{\frac{\sigma}{q}\left(x-\gamma_{j}-q \frac{\pi}{\sigma} k\right)} e^{i q \frac{\pi}{\sigma} k \mu}(j=1,2, \ldots, p)
$$


for every $\mu$ from $I_{0}$. Each periodic (its period is equal to the length of $I_{0}$ ) function $h_{j}$ is a smooth function inside the interval $I_{0}$ and a piece-wise smooth one on the real axis. Therefore, the Fourier series (2.12) converges to the function $h_{j}$ boundedly on the whole real axis (not only on $I_{0}$ ). From (2.6) and (2.12) it follows

$$
\begin{aligned}
e^{i \lambda x}= & \sum_{1 \leq j \leq p} \sum_{k \in \mathbb{Z}} \frac{D_{j}(x)}{D} \frac{\sin \frac{\sigma}{q}\left(x-\gamma_{j}-q \frac{\pi}{\sigma} k\right)}{\frac{\sigma}{q}\left(x-\gamma_{j}-q \frac{\pi}{\sigma} k\right)} \\
& \quad \times e^{i\left(q \frac{\pi}{\sigma} k+\gamma_{j}\right) \lambda} \text { for } \lambda \in E=\bigcup_{1 \leq n \leq p} I_{n}
\end{aligned}
$$

The series in (2.13) converges boundedly for every $\lambda \in \mathbb{R}$. (However, the equality in (2.13) holds only for $\lambda \in E$.) The logic in the previous consideration is rather broken. We showed that if the exponent $e^{i \lambda x}$ is representable on $E$ in the form (2.6), then this exponent expands into the series (2.13) on $E$. However, all our reasoning is invertible, and the equality (2.13) really holds. Analogously, if $q$ is even, we get the equality

$$
\begin{aligned}
e^{i \lambda x}= & \sum_{i \leq j \leq p} \sum_{k \in \mathbb{Z}} \frac{D_{j}(x)}{D}(-1)^{k} \frac{\sin \frac{\sigma}{q}\left(x-\gamma_{j}-q \frac{\pi}{\sigma} k\right)}{\frac{\sigma}{q}\left(x-\gamma_{j}-q \frac{\pi}{\sigma} k\right)} \\
& \times e^{i\left(q \frac{\pi}{\sigma} k+\gamma_{j}\right) \lambda} \text { for } \lambda \in E=\bigcup_{1 \leq n \leq p} \cdot I_{n}
\end{aligned}
$$

In formulas (2.13) and (2.14) $x$ is an arbitrary complex number. Now let $f$ be a function from the space $L^{2}(\mathbb{R})$, and let the spectrum of $f$ be contained in the set $E$, that is $f(x)=\int_{E} \varphi(\lambda) e^{i \lambda x} d x$. Inserting herein instead of the exponent $e^{i \lambda x}$ its expansion (2.13) or (2.14) on $E$ (the sum of the series (2.13), (2.14) outside of $E$ is not important because the function $\varphi$ vanishes outside of $E$ ) and changing the order of summation and integration, we get the formula

$$
f(x)=\sum_{1 \leq j \leq p} \sum_{k \in \mathbb{Z}} S_{j, k}(x) f\left(x_{j, k}\right)
$$

where

$$
x_{j, k}=q \frac{\pi}{\sigma} k+\gamma_{j} \quad(1 \leq j \leq p ; k \in \mathbb{Z})
$$

and

$$
S_{j, k}(x)=(-1)^{k(q-1)} \frac{D_{j}(x)}{D} \frac{\sin \frac{\sigma}{q}\left(x-x_{j, k}\right)}{\frac{\sigma}{q}\left(x-x_{j, k}\right)}
$$

Changing the order of summation and integration is permitted since the function $\varphi$ belongs to $L^{2}(E)$ and the series (2.14) converges boundedly. 
The formula (2.15) can be considered as an analogue of the sampling formula (1.3). The factor $(-1)^{k(q-1)}$ is introduced to treat simultaneously the cases of even and odd $q$. We shall prove later that the series in (2.15) converges both in $L^{2}(\mathbb{R})$ and locally uniformly in the complex plane.

Let us analyze the formulas (2.15) - (2.17). The set of points $\left\{x_{k, j}\right\}$ is decomposed into $p$ subsets, or $p$ 'series'. These series are enumerated by the index $j, j=$ $1,2, \ldots, p$. The $j$-th series consists of the sequence of equidistant points $x_{j, k}, x_{j, k}=\gamma_{j}+$ $\frac{q \pi}{\sigma} k$, where $k$ runs over the set $\mathbb{Z}$ of all integers. The set $\left\{S_{j, k}\right\}$ of the functions which figure up in the interpolation series (2.30) is also decomposed into $p$ series enumerated by the index $j, j=1,2, \ldots, p$. It follows from (2.10) and (2.11) that

$$
S_{j, k}\left(x_{j^{\prime}, k^{\prime}}\right)=\left\{\begin{array}{ll}
1 & \text { if } j=j^{\prime}, k=k^{\prime} \\
0 & \text { if }(j, k) \neq\left(j^{\prime}, k^{\prime}\right)
\end{array} .\right.
$$

In other words, each function $S_{j, k}$ vanishes at all the points $x_{j^{\prime}, k^{\prime}}$ of each 'alien' series (which is enumerated by an index $j^{\prime}, j^{\prime} \neq j$ ), and also at all the points $x_{j, k^{\prime}}$ of its 'own' series different from the point $x_{j, k}$. Thus, the series (2.15) is an interpolation one.

Let us see what is the spectrum of the function $S_{j, k}$. The spectrum of the function $\frac{\sin \frac{\sigma}{q}\left(x-x_{j, k}\right)}{\frac{\sigma}{q}\left(x-x_{j, k}\right)}$ is exactly the interval $I_{0}=\left[-\frac{\sigma}{q}, \frac{\sigma}{q}\right]$. As it is seen from (2.9), the function $D_{j}$ has the form

$$
D_{j}(x)=\sum_{1 \leq n \leq p} d_{n, j} e^{i \lambda_{n} x}
$$

where $d_{n, j}$ are some numbers - the algebraic complements of the $(n, j)$-th entries of the matrix. $\left\|e^{i \lambda_{n} \gamma_{j}}\right\|_{\substack{1 \leq n \leq p \\ 1 \leq j \leq p}}$ Thus

$$
S_{j, k}(x)=(-1)^{k(q-1)} \sum_{1 \leq n \leq p} \frac{d_{n, j}}{D} e^{i \lambda_{n} x} \frac{\sin \frac{\sigma}{q}\left(x-x_{j, k}\right)}{\frac{\sigma}{q}\left(x-x_{j, k}\right)}
$$

Hence, the spectrum of the function $S_{j, k}$ is contained in the set $\bigcup_{1 \leq n \leq p}\left(\lambda_{n}+I_{0}\right)$. As $\dot{\lambda_{n}}+I_{0}=I_{n}$, the spectrum of the function $S_{j, k}$ is contained in the set $E$. This spectrum coincides with the set $E$ if all the numbers $d_{j, n}$ are different from zero. Thus, each term of the series (not only its whole sum) has the spectrum contained in $E$.

Let us clarify whether the interpolation (2.15) is free. Let $\left\{c_{j, k}\right\}$ be an arbitrary sequence of complex numbers satisfying the condition $\sum_{1 \leq i \leq p} \quad \sum_{k \in \mathbb{Z}}\left|c_{j, k}\right|^{2}<$ $\infty$ or, what is the same, the condition

$$
\sum_{k \in \mathbb{Z}}\left|c_{j, k}\right|^{2}<\infty \quad(j=1,2, \ldots, p) .
$$


Then the series

$$
f_{c}(x)=\sum_{1 \leq j \leq p} \sum_{k \in \mathbb{Z}} c_{j, k} S_{j, k}(x)
$$

converges locally uniformly in $\mathbb{C}$ : from (2.16) - (2.17) it follows that

$$
\left|S_{j, k}(z)\right|^{2} \leq A \frac{e^{2 \sigma|\operatorname{Im} z|}}{1+\left|z-x_{j, k}\right|^{2}} \quad \text { for all } z \in \mathbb{C}
$$

where $A$ is a constant independent on $z$. Hence,

$$
\sum_{j, k}\left|S_{j, k}(z)\right|^{2} \leq B e^{2 \sigma|\operatorname{Im} z|}
$$

From the locally uniform convergence of the series (2.21) it follows that $f_{c}$ is an entire function, and from (2.20) and (2.22) it follows that $f_{c}$ is an entire function of an exponential type not exceeding $\sigma$. From (2.18) and (2.21) the interpolation condition

$$
f_{c}\left(x_{j, k}\right)=c_{j, k} \quad(1 \leq j \leq p ; k \in \mathbb{Z})
$$

follows. Let us show that $f_{c} \in L^{2}(\mathbb{R})$, and that the inequality

$$
\int_{\mathbb{R}}\left|f_{c}(x)\right|^{2} d x \leq \frac{M^{2}}{D^{2}} \sum_{1 \leq j \leq p} \sum_{k \in \mathbb{Z}}\left|c_{j, k}\right|^{2}
$$

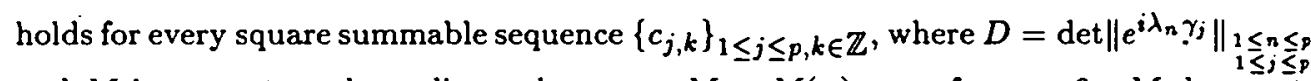
and $M$ is a constant depending only on $\sigma, M=M(\sigma)<\infty$ for $\sigma>0$. $M$ does not depend on the sequence $\left\{c_{j, k}\right\}$. It is sufficient to prove this inequality only for all finite sequences $\left\{c_{j, k}\right\}$. As $f_{c}$ is an entire function of exponential type not exceeding $\sigma$, the inequality of Plancherel-Pólya [27]

$$
\int_{\mathbb{R}}\left|f_{c}(x)\right|^{2} d x \leq e^{2 \sigma\left|y_{0}\right|} \int_{\mathbb{R}}\left|f_{c}\left(x+i y_{0}\right)\right|^{2} d x
$$

is satisfied for every $y_{0} \in \mathbb{R}$. (See also [2, item 6.7] or [32, Chapter 2, Theorem 16].) Hence, it is enough to prove that the inequality

$$
\int_{\mathbb{R}}\left|f_{c}\left(x+i y_{0}\right)\right|^{2} d x \leq \frac{M^{2}\left(\sigma, y_{0}\right)}{D^{2}} \sum_{1 \leq j \leq p} \sum_{k \in \mathbb{Z}}\left|c_{j, k}\right|^{2}
$$

holds for some real $y_{0}$ where $M\left(\sigma, y_{0}\right)<\infty$ is a constant not depending on $z$. (The inequality (2.25) with $y_{0} \neq 0$ can be proved easier than this one with $y_{0}=0$.) The function $f_{c}$ is a sum: $f_{c}(z)=\sum_{1 \leq j \leq p} f_{c, j}(z)$, where

$$
f_{c, j}(z):=\sum_{k \in \mathbb{Z}} c_{j, k} S_{j, k}(z) \text {. }
$$


It is enough to prove an inequality of type (2.25) for each function $f_{c, j}, 1 \leq j \leq p$.

Lemma 2.1: Let $\left\{t_{k}\right\} \subset \mathbb{R}$ be a sequence satisfying the separation condition

$$
\inf _{k^{\prime} \neq k^{\prime \prime}}\left|t_{k^{\prime}}-t_{k^{\prime \prime}}\right|=\delta>0
$$

$\left\{\xi_{k}\right\} \in \mathbb{C}$ be a finite sequence and $y_{0} \in \mathbb{R}$ be a non-zero real number. Then

$$
\int_{\mathbb{R}}\left|\sum_{k \in \mathbb{Z}} \frac{\xi_{k}}{x-t_{k}-i y_{0}}\right|^{2} d x \leq N_{1}^{2}\left(y_{0}, \delta\right) \sum_{k \in \mathbb{Z}}\left|\xi_{k}\right|^{2}
$$

where $N_{1}=N_{1}\left(y_{0}, \delta\right)$ is a constant not depending on the sequence $\left\{\xi_{k}\right\}, N_{1}\left(y_{0}, \delta\right)<\infty$ for $y_{0} \neq 0, \delta>0$.

The operator $\left\{\xi_{k}\right\}_{k \in \mathbb{Z}} \longrightarrow\left(\sum_{k \in \mathbb{Z}} \frac{\xi_{k}}{x-t_{k}-i y_{0}}\right)_{x \in \mathbb{R}}$ is a discrete-continuous analogue of the Hilbert transform ( $y_{0}$ is some parameter). The inequality (2.28) shows that for $y_{0} \neq 0$ this operator is a bounded linear one from $\ell^{2}$ to $L^{2}(\mathbb{R})$.

We prove Lemma 2.1 later. Now we show how the inequality (2.25) follows from the inequality (2.28). The equality (2.19) can be rewritten in the form

$$
\begin{aligned}
S_{j, k}(x)=(-1)^{k q} & \frac{q}{\sigma} \sin \frac{\sigma}{q}\left(z-\gamma_{j}\right) \\
& \times\left(\sum_{1 \leq n \leq p} \frac{d_{n, j}}{D} e^{i \lambda_{n} x}\right) \frac{1}{x-\gamma_{j}-k \frac{q \pi}{\sigma}} \quad(x \in \mathbb{C}) .
\end{aligned}
$$

From (2.26) and (2.29) it follows that

$$
\left|f_{c, j}\left(x+i y_{0}\right)\right| \leq \frac{N}{D}\left|\sum_{k \in \mathbb{Z}} \frac{c_{j, k}(-1)^{k q}}{x-\left(\gamma_{j}+k \frac{q \pi}{\sigma}-i y_{0}\right)}\right| \quad\left(x, y_{0} \in \mathbb{R}, y_{0} \neq 0\right)
$$

where $N=N\left(y_{0}, \sigma\right)$ is a constant not depending on the sequence $\left\{c_{j, k}\right\}, N\left(y_{0}, \sigma\right)<\infty$ for $y_{0} \neq 0, \sigma<\infty$. It results from $(2.30)$ that

$$
\int_{\mathbb{R}}\left|f_{c, j}\left(x+i y_{0}\right)\right|^{2} d x \leq \frac{N^{2}}{D^{2}} \int_{\mathbb{R}}\left|\sum_{k \in \mathbb{Z}} \frac{c_{j, k}(-1)^{k q}}{x-\left(\dot{\gamma}_{j}+k \frac{q \pi}{\sigma}-i y_{0}\right)}\right|^{2} d x
$$

The sequence $\left\{t_{k}\right\}=\left\{\gamma_{j}+k \frac{q \pi}{\sigma}\right\} \quad(k \in \mathbb{Z}, \quad j$ is a parameter) satisfies the separation condition (2.27) with $\delta=. \frac{g \pi}{2 \sigma}$. From (2.28) (with $\xi_{k}=(-1)^{k q} c_{j, k}$ and $t_{k}=\gamma_{j}+k \frac{q \pi}{\sigma}$ ) we obtain the inequality

$$
\int_{\mathbb{R}}\left|f_{c, j}\left(x+i y_{0}\right)\right|^{2} d x \leq \frac{M^{2}}{D^{2}} \sum_{k \in \mathbb{Z}}\left|c_{j, k}\right|^{2}
$$


where $M=N N_{1}$ does not depend on the sequence $\left\{c_{j, k}\right\}$. The deduction of the inequality (2.24) from the inequality (2.28) is finished.

We shall prove the inequality (2.28) later, after the formulation of the main theorem of this item. Later, we shall also prove the 'inverse' (relative to (2.24)) inequality

$$
m^{2} \sum_{1 \leq j \leq p ; k \in \mathbb{Z}}\left|c_{j, k}\right|^{2} \leq \int_{\mathbb{R}}\left|f_{c}(x)\right|^{2} d x
$$

where $m$ is a constant depending only on $\sigma, m=m(\sigma)>0$ for $\sigma<\infty$.

From the inequality (2.24) it follows immediately that (by condition $D \neq 0$ ) the series (2.21) converges in $L^{2}(\mathbb{R})$ and defines a function $f_{c}$ from $L^{2}(\mathbb{R})$ for every square

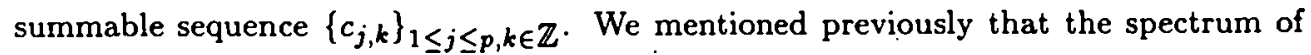
each term of the series (2.21) is contained in the set $E$. If the series (2.21) converges in $L^{2}(\mathbb{R})$, the spectrum of its sum is also contained in the set $E$.

Thus, we have proved the most of the assertions of the following

Theorem: Let $E$ be a subset of the interval $[-\sigma, \sigma]$ which has 'multiband' structure (2.1) - (2.2) as described above: $E$ is the union of $p$ regularly positioned intervals of the length $\frac{2 \sigma}{q}$ with the centres $\lambda_{n}, n=1,2, \ldots, p$ (see (2.1)), $p$ and $q$ are positive integers, $p<q$. Let $\gamma_{j}(j=1,2, \ldots, p)$ be real numbers satisfying the condition $D \neq 0$ where $D:=\operatorname{det}\left\|e^{i \lambda_{n} \gamma_{j}}\right\|_{\substack{1 \leq n \leq p \\ 1 \leq j \leq p}}$. Let $\left\{x_{j, k}\right\}_{1 \leq j \leq p, k \in \mathbb{Z}} \subset \mathbb{R}$ be the countable set which is defined in (2.16) and let $S_{j, k}(1 \leq j \leq p, k \in \mathbb{Z})$ be the functions defined in (2.17). Then:

i) Each function $f \in L^{2}(\mathbb{R})$ whose spectrum is contained in $E$ can be recovered from its values $\left\{f\left(x_{j, k}\right)\right\}$ at the sampling points $\left\{x_{j, k}\right\}_{1 \leq j \leq p, k \in \mathbb{Z}}$ by means of the interpolation series (2.15) which converges both in $L^{2}(\mathbb{R})$ and locally uniformly in the complex plane. The inequalities

$$
m^{2} \sum_{1 \leq j \leq p ; k \in \mathbb{Z}}\left|f\left(x_{j, k}\right)\right|^{2} \leq \int_{\mathbb{R}}|f(x)|^{2} d x \leq \frac{M^{2}}{D^{2}} \sum_{1 \leq j \leq p ; k \in \mathbb{Z}}\left|f\left(x_{j, k}\right)\right|^{2}
$$

hold where $m$ and $M$ are values depending only on $\sigma$ and not depending on $f$ :

$$
m=m(\sigma)>0, \quad M=M(\sigma)<\infty .
$$

ii). For an arbitrary square summable set $\left\{c_{j, k}\right\}_{1 \leq j \leq p, k \in \mathbb{Z}}$ of complex numbers the series (2.21) converges both in $L^{2}(\mathbb{R})$ and locally uniformly in the complex plane. The sum $f_{c}$ of this series is a function belonging to $L^{2}(\mathbb{R})$ whose spectrum is contained in $E$. The function $f_{c}$ satisfies the interpolation conditions (2.29). 
A part of the assertions of this theorem has been already proved. To finish the proof, we should prove the inequalities (2.32) and clarify the character of the convergence of the series.

Lemma 2.2: Let $\left\{t_{k}\right\}$ be a sequence of real numbers satisfying the separation condition (2.27), and let $\tau$ be a positive number. Let $w$ be a function from the Hardy class $H^{2}$ in the upper half-plane. Then the inequality

$$
m^{2} \sum_{k}\left|w\left(t_{k}+i \tau\right)\right|^{2} \leq \int_{\mathbb{R}}|w(x)|^{2} d x
$$

holds where $m$ is a constant not depending on the function $w$ ( $m$ depends only on $\tau$ and the constant $\delta$ occuring the separation condition (2.27)):

$$
m=m(\delta, \tau)>0 \quad \text { for } \tau \in(0, \infty), \delta>0 \text {. }
$$

Proof: Let $\rho=\min \left(\frac{\delta}{2}, \tau\right)$. Let $z_{k}=t_{k}+i \tau$, and $C_{k}=\left\{z:\left|z-z_{k}\right|<\rho\right\}$. Evidently the circles $C_{k}$ do not intersect and are contained in the strip $\Pi_{\rho}=\{z \in \mathbb{C}$ : $0<\operatorname{Im} z<2 \rho$ \} of the upper half-plane. Therefore, the inequality

$$
\sum_{k} \iint_{C_{k}}|w(x+i y)|^{2} d x d y \leq \iint_{\Pi_{p}}|w(x+i y)|^{2} d x d y
$$

holds. As the function $|w|^{2}$ is a subharmonic one, the inequalities

$$
\left|w\left(z_{k}\right)\right|^{2} \leq \frac{1}{\pi \rho^{2}} \iint_{C_{k}}|w(z)|^{2} d x d y \quad(k \in \mathbb{Z})
$$

are satisfied. Combining these inequalities, we obtain the inequality

$$
\pi \rho^{2} \sum_{k}\left|w\left(z_{k}\right)\right|^{2} \leq \iint_{\Pi_{\rho}}|w(x+i y)|^{2} d x d y .
$$

As for every function $w$ from the Hardy class $H^{2}$ the inequality $\int_{\mathbb{R}}|w(x+i y)|^{2} d x \leq$ $\int_{\mathbb{R}}|w(x)|^{2} d x$ holds, the inequality

$$
\iint_{\Pi_{\rho}}|w(x+i y)|^{2} d x d y \leq 2 \rho \int_{\mathbb{R}}|w(x)|^{2} d x
$$

holds as well. Comparing the inequalities (2.35) and (2.36), we get the inequality (2.34) with $m^{2}=\frac{\pi \rho}{2}$

Remark: Of course, Lemma 2.2 is a very special case of Carleson's theorem concerning the so-called Carleson measures. The Borel measure $\mu$ in the upper halfplane is called a Carleson measure, if the inequality

$$
\iint_{I m z>0}|w(z)|^{2} \mu(d z) \leq C \int_{\mathbb{R}}|w(x)|^{2} d x
$$


holds for every function $w$ from $H^{2}$ where $C<\infty$ is a constant not depending on $w$. This theorem is stated, for example, in [9, Chapter 2, Theorem 3.9]. If a real sequence $\left\{t_{k}\right\}$ satisfies the separation condition (2.27), then the sequence $\left\{z_{k}\right\}$ given by $\left\{z_{k}\right\}=\left\{t_{k}+i \tau, \tau>0\right\}$ generates a Carleson measure. However, the sequence $\left\{z_{k}\right\}$ is a very special one, and our proof of Lemma 2.2 is much easier than any other proof of the general Carleson theorem.

Proof of the left of the inequalities (2.32). Choose some number $\tau, \tau>0$, and fix it. If a function $f$ satisfying the assumptions of the theorem is given, we consider the function $w$,

$$
w(z)=f(z-i \tau) e^{i \sigma(z-i \tau)} \quad(\operatorname{Im} z>0)
$$

We consider only the restriction of $w$ on the upper half plane. It follows from the assumptions on the function $f$ that the function $w$ belongs to the Hardy class $H^{2}$ in the upper half plane. In accordance with Lemma 2.1 we have the inequality

$$
m_{1} \sum_{k}\left|w\left(t_{k}+i \tau\right)\right|^{2} \leq \int_{\mathbb{R}}|w(x)|^{2} d x
$$

with $m_{1}>0$ not depending on $w$. Taking into account (2.37) we obtain the inequality

$$
m_{1} \sum_{k}\left|f\left(t_{k}\right)\right|^{2} \leq e^{2 \sigma \tau} \int_{\mathbb{R}}|f(x-i \tau)|^{2} d x .
$$

As $f$ is an entire function of exponential type not exceeding $\sigma$, the inequality

$$
\int_{\mathbb{R}}|f(x-i \tau)|^{2} \leq e^{2 \sigma \tau} \int_{\mathbb{R}}|f(x)|^{2} d x
$$

holds. Comparing the inequalities (2.38) and (2.39), we obtain the inequality

$$
m_{1}(\tau, \delta) \sum_{k}\left|f\left(t_{k}\right)\right|^{2} \leq e^{4 \sigma \tau} \int_{\mathbb{R}}|f(x)|^{2} d x
$$

For each $j, j=1,2, \ldots, p$, the sequence $\left\{x_{j, k}\right\}_{k \in \mathbb{Z}}$ satisfies the separation condition (2.27) with $\delta=\frac{q \pi}{\sigma}$. Therefore, for each such $j$ the inequality

$$
\mu \sum_{k}\left|f\left(x_{j, k}\right)\right|^{2} \leq e^{4 \sigma \tau} \int_{\mathbb{R}}|f(x)|^{2} d x
$$

holds with a constant $\mu>0$ not depending on $f, \mu=\mu(\sigma, \tau)$. Summing up all these inequalities over $j$, we get the inequality

$$
m \cdot \sum_{1 \leq j \leq p ; k \in \mathbb{Z}}\left|f\left(x_{j, k}\right)\right|^{2} \leq \int_{\mathbb{R}}|f(x)|^{2} d x
$$


with a constant $m$ not depending on $f, m=m(\sigma)>0$ for $\sigma<\infty$

Remark: In the proof of the inequality (2.40) we have not used any special structure of the spectrum of the function $f$. We used only that the spectrum of $f$ is contained in the interval $[-\sigma, \sigma]$ and hence $f$ is an entire function of exponential type not exceeding $\sigma$. Inequalities of the form (2.40) are well-known. Firstly, such an inequality has been given by M. Plancherel and G. Pólya (see [27, especially items 27-31]). This result and its proof are reproduced, for example, in [2, pp. 97-103] and in [32, Chapter 2, item 3, especially Theorem 17 there].

Proof of Lemma 2.1: Perhaps the simplest way to prove the inequality (2.28) is via using duality reasonings. Let $y_{0}>0$. For every finite sequence $\xi=\left\{\xi_{k}\right\}_{k \in \mathbb{Z}}$ the function

$$
g_{\xi}(z)=\sum_{k \in \mathbb{Z}} \frac{\xi_{k}}{z-t_{k}-i y_{0}}
$$

of the complex variable $z$ belongs to the Hardy class $H^{2}$ in the upper half-plane. As $H^{2}$ is a Hilbert space, the dual space can be identified with the space $H^{2}$ itself in standard way. Hence, for every function $g \in H^{2}$ the inequality

$$
\int_{\mathbb{R}}|g(x)|^{2} d x=\sup \left|\int_{\mathbb{R}} h(x) \overline{g(x)} d x\right|^{2}
$$

holds, where supremum is taken over all normalized functions $h \in H^{2}: \int_{\mathbb{R}}|h(x)|^{2} d x=$ 1. Applying the relation (2.42) to $g=g_{\xi}$ of the form (2.41) we obtain

$$
\int_{\mathbb{R}}\left|\sum_{k \in \mathbb{Z}} \frac{\xi_{k}}{x-t_{k}-i y_{0}}\right|^{2} d x=\sup \left|\sum_{k \in \mathbb{Z}} \bar{\xi}_{k} \int_{\mathbb{R}} h(x) \frac{1}{x-t_{k}-i y_{0}} d x\right|^{2},
$$

According to Cauchy's formula,

$$
\int_{\mathbb{R}} h(x) \frac{1}{x-t_{k}-i y_{0}} d x=2 \pi i h\left(t_{k}+i y_{0}\right)
$$

Hence,

$$
\int_{\mathbb{R}}\left|\sum_{k \in \mathbb{Z}} \frac{\xi_{k}}{x-t_{k}-i y_{0}}\right|^{2} d x=4 \pi^{2} s u p\left|\sum_{k \in \mathbb{Z}} \dot{\overline{\xi_{k}}} \dot{h}\left(t_{k}+i y_{0}\right)\right|^{2},
$$

where supremum is taken over all normalized functions $h$ from $H^{2}$. As

$$
\left|\sum_{k \in \mathbb{Z}} \bar{\xi}_{k} h\left(t_{k}+i y_{0}\right)\right|^{2} \leq\left(\sum_{k \in \mathbb{Z}}\left|\xi_{k}\right|^{2}\right)\left(\sum_{k \in \mathbb{Z}}\left|h\left(t_{k}+i y_{0}\right)\right|^{2}\right),
$$


we obtain from (2.43) that

$$
\int_{\mathbb{R}}\left|\sum_{k} \frac{\xi_{k}}{x-t_{k}-i y_{0}}\right|^{2} d x \leq\left(\sum_{k}\left|\xi_{k}\right|^{2}\right)\left(\sup _{h} \sum_{k}\left|h\left(t_{k}+i y_{0}\right)\right|^{2}\right) .
$$

According to Lemma 2.2, the inequality

$$
\sum_{k \in \mathbb{Z}}\left|h\left(t_{k}+i y_{0}\right)\right|^{2} \leq C\left(\delta, y_{0}\right) \int_{\mathbb{R}}|h(x)|^{2} d x
$$

holds for every $h$ belonging to $H^{2}$ where $C\left(\delta, y_{0}\right)$ depends only on $y_{0}$ and $\delta$ (from the separation condition (2.27)). Combining inequalities (2.44) and (2.45) and taking into account the normalizing condition $\int_{\mathbb{R}}|h(x)|^{2} d x=1$ we obtain the inequality (2.28)

Now we are completing the proof of the theorem of this item. Let the function $f$ satisfy the condition of the theorem. We have proved that the inequality (2.40) holds. Thus, the condition

$$
\sum_{1 \leq j \leq p} \sum_{k \in \mathbb{Z}}\left|f\left(x_{j, k}\right)\right|^{2}<\infty
$$

is satisfied. We have proved that the function $f$ is representable in the form (2.15). Thus, $f$ has the form $f_{c}$ of $(2.21)$, with $c_{j, k}=f\left(x_{j, k}\right)$. Of course, the condition $(2.46)$ is the same as the condition $\sum_{k \in \mathbb{Z}}\left|c_{j, k}\right|^{2}<\infty \quad(j=1,2, \ldots, p)$. For any function $f_{c}$ (with square summable $C=\left\{c_{j, k}\right\}$ ) we have proved that the series (2.21) converges both in $L^{2}(\mathbb{R})$ and locally uniformly in $\mathbb{C}$. We have also proved the inequality (2.24). However, for $c_{j, k}=f\left(x_{j, k}\right)$ this inequality goes over into the right inequality (2.32), and the series (2.21) goes over into the series (2.15). This completes the proof of the theorem

\section{Remarks regarding the theorem of the previous section}

In this section some remarks are given in which we supplement and specify results of the previous section.

Remark 1: In particular, we have proved the following uniqueness theorem: If $f \in L^{2}(\mathbb{R})$, its spectrum is contained in the set $E$ of described structure, the point

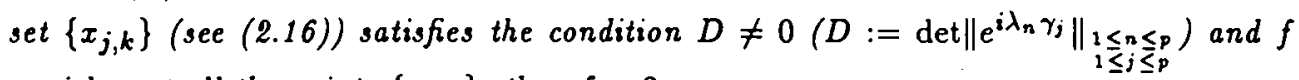
vanishes at all the points $\left\{x_{j, k}\right\}$, then $f \equiv 0$.

Remark 2: It would be possible starting from a set $E$ with 'multi-band' structure, to construct functions $S_{j, k}$ via formula (2.17) and then, starting from a function $f \in$ 
$L^{2}(\mathbb{R})$ with spectrum in $E$, to construct the series (2.15). From properties (2.18) of the functions $S_{j, k}$ it follows immediately that the sum of these series coincides with the original function $f$ at all the points $x_{j, k}$. However, all the same we cannot do without reasoning using the expansion of the exponent $e^{i \lambda x}$ into a Fourier series on $E$ since we do not dispose of any independent proof of the uniqueness theorem.

Remark 3: Let us show, that if $\lambda_{1}, \lambda_{2}, \ldots, \lambda_{n}$ are given real numbers, $\lambda_{1}<\lambda_{2}<$ $\ldots<\lambda_{n}$, then there exist real numbers $\gamma_{1}, \gamma_{2}, \ldots, \gamma_{n}$ which satisfy the condition $D \neq 0$, where

$$
D=D\left(\lambda_{1}, \ldots, \lambda_{n} ; \gamma_{1}, \ldots, \gamma_{n}\right):=\operatorname{det}\left\|e^{i \lambda_{n} \gamma_{j}}\right\|_{\substack{1 \leq n \leq p \\ 1 \leq j \leq p}} .
$$

For any number $\rho>0$, we put $\gamma_{j}(\rho)=\rho(j-1), j=1,2, \ldots, p$. Denote $\zeta_{n}(\rho)=$ $e^{i \lambda_{n} \rho}, n=1,2, \ldots, p$. Then $\Phi(\rho)=D\left(\lambda_{1}, \ldots, \lambda_{n} ; \gamma_{1}(\rho), \ldots, \gamma_{n}(\rho)\right)$ has the form

$$
\Phi(\rho)=\operatorname{det}\left[\begin{array}{cccc}
1 & 1 & \ldots & 1 \\
\zeta_{1} & \zeta_{2} & \ldots & \zeta_{n} \\
\cdot & \cdot & \cdot & \cdot \\
\zeta_{1}^{p-1} & \zeta_{2}^{p-1} & \ldots & \zeta_{n}^{p-1}
\end{array}\right] .
$$

This is a Vandermonde determinant. It can be calculated explicitly:

$$
\Phi(\rho)=\prod_{1 \leq j<k \leq n}\left(\zeta_{k}(\rho)-\zeta_{j}(\rho)\right)
$$

If all the numbers $\zeta_{k}$ are pairwise different, this determinant does not vanish. The coincidence $\zeta_{m}(\rho)=\zeta_{n}(\rho), \quad m \neq n$, means that $e^{i\left(\lambda_{m}-\lambda_{m}\right) \rho}=1$. As $\lambda_{m} \neq \lambda_{n}, \mid \lambda_{m}-$ $\lambda_{n} \mid \leq 2 \sigma \frac{q-1}{q}$, the equality $\Phi(\rho)=0$ is impossible for $\rho$ sufficiently small, namely, for $0<\rho<\frac{\pi}{\sigma} \frac{q}{q-1}$. Thus, it is possible to choose numbers $\gamma_{1}, \ldots, \gamma_{n}$ in such a way that the condition $D\left(\lambda_{1}, \ldots, \lambda_{n} ; \gamma_{1}, \ldots, \gamma_{n}\right) \neq 0$ is fulfilled. For example, we can choose $\gamma_{j}$ of the form $\gamma_{j}(\rho)=\rho(j-1)$ with $\rho$ satisfying $0<\rho<\frac{\pi}{\sigma} \frac{q}{q-1}$.

For fixed $\lambda_{1}, \ldots, \lambda_{n}$ the function $D\left(\lambda_{1}, \ldots, \lambda_{n} ; \gamma_{1}, \ldots, \gamma_{n}\right)$ is an entire function of the variables $\gamma_{1}, \ldots, \gamma_{n}$. This entire function does not vanish identically. Thus, the set of 'forbidden' real $\gamma_{j}$ (satisfying the condition $D\left(\lambda_{1}, \ldots, \lambda_{n} ; \gamma_{1}, \ldots, \gamma_{n}\right)=0$ ) is contained in a real analytic manifold whose real codimension equals one and which is 'small' in this sense.

Remark 4: Let the set $E$ have the above-described 'multi-band' structure. The analysis of our reasoning above shows that the system of the exponential functions $\left\{e^{i \lambda x_{j, k}}\right\}_{1 \leq j \leq p ; k \in \mathbb{Z}}$, with $\left\{x_{j, k}\right\}$ defined in (2.16) is a Riesz basis in the space $L^{2}(E)$ 
(necessary information concerning Riesz bases can be found, for example, in [10, 16, 25, $32])$. On the contrary, let the system $\left\{e^{i \lambda x_{m}}\right\}$ be a Riesz basis in the space $L^{2}(E)$. Let $\left\{\varphi_{j}\right\}$ be a biorthogonal system:

$$
\int_{E} \varphi_{j}(\lambda) e^{i \lambda x_{m}} d \lambda=\delta_{j, m}
$$

Put

$$
S_{j}(x)=\int_{E} \varphi_{j}(\lambda) e^{i \lambda x} d \lambda \quad(x \in \mathbb{C})
$$

The biorthogonality condition (3.1) implies

$$
S_{j}\left(x_{m}\right)=\delta_{j, m}
$$

Let

$$
e^{i \lambda x}=\sum_{k} c_{k}(x) e^{i \lambda x_{k}} \quad(\lambda \in E)
$$

be the expansion of the exponential function $\left\{e^{i \lambda x}\right\}_{\lambda \in E}$ into the Fourier series for the basis $\left\{e^{i \lambda x_{k}}\right\}$ ( $x$ is considered as a parameter), where $\left\{c_{k}(x)\right\}_{k}$ are the corresponding Fourier coefficients. Multiplying equality (3.4) by the function $\varphi_{j}$, integrating over $E$ and taking into account (3.1) and (3.2), we get $S_{j}=c_{j}$. Now let $f \in L^{2}(\mathbb{R})$ with spectrum contained in $E$, and let $\varphi$ be its Fourier transform. Multiplying (3.4) by $\varphi$ and integrating over $E$, we get the equality

$$
f(x)=\sum_{k} S_{k}(x) f\left(x_{k}\right) \quad(x \in \mathbb{C}) .
$$

It follows from condition (3.3) that the series (3.5) is an interpolation one. As the system $\left\{e^{i \lambda x_{m}}\right\}$ is a Riesz basis, the interpolation (3.5) is free, and the inequalities

$$
m \sum_{k}\left|f\left(x_{k}\right)\right|^{2} \leq \int_{\mathbb{R}}|f(x)|^{2} d x \leq M \sum_{k}\left|f\left(x_{k}\right)\right|^{2}
$$

hold for every $f$ with spectrum contained in $E$, where $m>0$ and $M<\infty$ are constants not depending on $f$.

Thus, the sampling theorem for functions with spectrum in $E$ is equivalent (in some sense) to the problem of constructing a Riesz basis $\left\{e^{i \lambda x_{m}}\right\}$ in the space $L^{2}(E)$.

Remark 5: We established that for a set $E$ of the above-described 'multi-band' structure there exist sets of samplings for which the sampling theorem is true. Or, in other words, in $L^{2}(E)$ there exist bases of exponents $\left\{e^{i \lambda x_{m}}\right\}$. We have constructed a concrete example of such a set $\left\{x_{m}\right\}$. This set is the union of $n$ arithmetical progressions 
of real points (with the same spacing). Of course, there exist sets of samplings for which the sampling theorem is true and which are not representable as a union of arithmetical progressions. Such sets of samplings can be constructed using various theorems on perturbations of Riesz bases (see, for example, [10]).

Remark 6: If the spectrum of a function $f$ is the interval $[-\sigma, \sigma]$ (or if this spectrum is contained in $[-\sigma, \sigma])$, then for a set $X$ of samplings of the form $X=$ $\cup_{k \in \mathbb{Z}}\left\{\frac{k \pi}{\sigma}\right\}$ the sampling theorem is true. A set $X$ of this form has density $d(X)=\frac{\sigma}{\pi}$. As usual, the density $d(X)$ of some discrete set $X$ is defined as $d(X)=\lim _{r \rightarrow \infty} \frac{n_{X}(r)}{r}$, where $n_{X}=n_{X}(r)$ is the so-called counting function of $X-$ the number of points of $X$ which are contained in the disc $|z| \leq r$. On the other side, the Lebesgue measure $\operatorname{mes}(E)$ of the set $E=[-\sigma, \sigma]$ is equal to $2 \sigma: \operatorname{mes}(E)=2 \sigma$. So, for the set $E=[-\sigma, \sigma]$ and for the set $X=\cup_{k \in \mathbb{Z}}\left\{\frac{k \pi}{\sigma}\right\}$ the relation

$$
\operatorname{mes}(E)=2 \pi d(X)
$$

holds. Further, if $E$ has the above-defined multi-band structure (2.1) - (2.2) and if $X=\cup\left\{\dot{x}_{j, k}: 1 \leq j \leq p, k \in \mathbb{Z}\right\}$ with $\left\{x_{j, k}\right\}$ defined in (2.16), then for these $X$ and $E$ we have $\operatorname{mes}(E)=2 \sigma \frac{p}{q}$ and $d(X)=\frac{\sigma}{\pi} \frac{p}{q}$. As before, the equality (3.6) is true. We established that for any function which spectrum is contained in such $E$ and for such $X$ the sampling theorem is true (by additional condition $D \neq 0$ ).

Generalizing, we can formulate the following principle:

If the spectrum of a function $f$ is contained in a compact set $E$ whose Lebesgue measure mes $(E)$ is positive, a discrete subset $X \subset \mathbb{R}$ has density $d(X)$, and if the relation (9.6)-issatisfied, then $f$ can be recovered from its values at the points of $X$, and the inequalities $m \sum_{x \in X}|f(x)|^{2} \leq \int_{\mathbb{R}}|f(x)|^{2} d x \leq M \sum_{x \in X}|f(x)|^{2}$ hold true $(m>0$ and $M<\infty$ do not depend on $f)$. In other words, this principle states that by condition (9.6) the set $\left\{e^{i \lambda x}\right\}_{x \in X}$ forms a Riesz basis in $L^{2}(E)$.

Of course, it is only a principle but.not a rigorous-result. "The exact formulation must firstly embrace the separation condition

$$
\text { in } f\left|x^{\prime}-x^{\prime \prime}\right|>0^{\circ} \quad\left(x^{\prime}, x^{\prime \prime} \in X \text { with } x^{\prime} \neq x^{\prime \prime}\right)
$$

and secondly, a condition which should express uniformity of distribution of the points of $X$. Such 'uniformity' condition can be formulated in terms of the function $w_{h}$,

$$
w_{h}(t)=\sum_{x \in X} \frac{1}{t-x+i h} \quad(t \in \mathbb{R})
$$


where $h \in \mathbb{R}, \quad h \neq 0$ is a (not essential) parameter. The function $w_{h}$ can be considered as some analogue of the Hilbert transform of the counting measure $d n_{x}$.

If $E=[-\sigma, \sigma]$, necessary and sufficient conditions are known for the set $\left\{e^{i \lambda x}\right\}_{x} \in X$ to be a Riesz basis in $L^{2}(E)$ (see [16]). These conditions embrace the condition (3.6) giving the value for density $d(X)$ of the set $X$, the separation condition (3.7) and (what is most difficult) some condition on the function $w_{h}$ (see (3.8)).

If the compact set $E$ is not an interval, no general conditions on a set $X \subseteq \mathbb{R}$ are known for the appropriate set of exponents to be a Riesz basis in $L^{2}(E)$. However, we think that in any case such conditions must include a condition like (3.6). If the set $E$ has the above-described multi-band structure (it is a finite union of regularly positioned intervals having equal or (what is the same!) commensurable lengths), then there exists bases of exponents in $L^{2}(E)$. We are not able to give any general conditions, but we are able at least to construct some family of such bases in $L^{2}(E)$. These bases correspond to the set $X$ of the form $X=\cup\left\{x_{j, k}: 1 \leq j \leq p, k \in \mathbb{Z}\right\}$ with $\left\{x_{j, k}\right\}$ defined in (2.16). For this set $X$ the density condition (3.6) and the separation condition (3.7) (remind that all the $\gamma_{j}$ are pairwise different) and the uniformity condition are satisfied. However, for a multi-band set $E$ only these conditions do not ensure the basis property. Besides these conditions, we need the additional condition $D \neq 0$

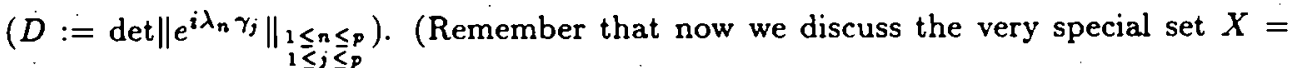
$\cup\left\{x_{j, k}: 1 \leq j \leq p, k \in \mathbb{Z}\right\}$ with $\left\{x_{j, k}\right\}$ defined in (2.16).) It is not difficult to get a more general result about the set $E$ which is the finite union of disjoint intervals having commensurable lengths and general (not necessarily regular) position on the real axis. However, our method does not work for the set $E$ : which is the finite union of disjoint intervals with noncommensurable lengths. We do not know now if there exists a Riesz basis of exponents in $L^{2}(E)$ for such a set $E$. All the more, we do not know whether there exists a Riesz basis of exponents in $L^{2}(E)$ in the case that the set $E$ is some Cantor-like set of positive measure. This will be the subject of our further investigations.

Remark 7: In his paper [21] H.J. Landau constructed an example of a discrete subset $X=\left\{x_{k}\right\}_{k \in \mathbb{Z}}$ of $\mathbb{R}$ and a multiband set $E$ such that the system of exponentials $\left\{e^{i \lambda x_{k}}\right\}_{k \in \mathbb{Z}}$ is complete in the space $L^{2}(E), d(X)=1$, and mes $E$ can be arbitrary large. Thus, the condition (3.6) is not necessary for the completeness of a system of exponentials in $L^{2}(E)$. In this example a set $E$ can be in particular a finite union of regularly positioned intervals of the same length.

On the other side, from Theorems 1 and 2 of the paper [22] by H.J. Landau it follows that condition (3.6) is necessary for the fact that the system of exponentials 
$\left\{e^{i \lambda x_{k}}\right\}_{k \in \mathbb{Z}}$ is a Riesz basis in the space $L^{2}(E)$ where $E$ is a multiband set. No additional restrictions concerning lenghts or positions of intervals the union of which is the set $E$ are supposed. (This formulation is weaker than the more precise result in [22].)

Remark 8: For a multiband set $E$ which is a finite union of regularly positioned intervals of the same length we constructed a discrete set $X=\left\{x_{k}\right\}$ of sampling points in the following way: we partition the integers into disjoint subsets, shift each subset by an amount, and let $\left\{x_{k}\right\}$ be the set which consist of these points. In papers $[1,3,6$, $7,14]$ an other construction of a set $X=\left\{x_{k}\right\}$ of sampling points is used. Let $E$ be a closed subset of $\mathbb{R}$, and let $\rho$ be a positive number which satisfy the condition

$$
\operatorname{mes}((E+2 \pi k \rho) \cap E)=0 \quad(\text { for all } k \in \mathbb{Z} \backslash\{0\})
$$

where $E+2 \pi k \rho=\{\lambda+2 \pi k \rho: \lambda \in E\}$. Then any function $f$ with Fourier transform $\rho$ vanishing outside $E$ can be reconstructed from samples taken at a rate $\varphi$, by means of the formula

$$
f(t)=\sum_{k \in \mathbb{Z}} f\left(\frac{k}{\rho}\right) S_{E}\left(t-\frac{k}{\rho}\right)
$$

where

$$
S_{E}(t)=\frac{1}{m e s E} \int_{E} e^{i t \lambda} d \lambda \quad(T \in \mathbb{R}) .
$$

Various aspects of the representation $(3.10)-(3.11)$ (by the condition (3.9)) are discussed in $[1,3,6,7,14]$.

Remark 9: In Goldman's book [11] functions $f \in L^{2}(\mathbb{R})$ are considered which spectrum is contained in a set of the form $E=\left[-\omega_{2},-\omega_{1}\right] \cup\left[\omega_{1}, \omega_{2}\right]$ where $0<w_{1}<$ $w_{2}<\infty$. In $[11, \S 2.3]$, an assertion on such functions has been proved called 'Sampling Theorem'. However, the interpolation formula obtained in [11] differs from our sampling formula (2.15) - (2.17). In Goldman's interpolation formula there figure up not only the values of the function $f$ itself, but also the values of its Hilbert transform. Furthermore, Goldman's sampling formula has been established only for functions with two-band spectrum (with bands of equal length). It is not clear to us how to extend this result of Goldman to a more general case. vskip $.2 \mathrm{~cm}$

Remark 10: After the initial version of this paper was submitted, the authors sent a preprint to J.R. Higgins and received as a response a preprint of the latter's paper [15]. It turned out that these two papers overlap essentially. However, our treatment is more general than the 'harmonic signals' treated in Section 5 of paper [15]. 


\section{References}

[1] BEATY, M.G., and M.M. DODSON: The distribution of sampling rates for signals with equally uide, equally spaced spectral bands. SIAM J. Appl. Math. (to appear).

[2] BoAs, R.F.: Entire Functions. New York and London: Academic Press 1954.

[3] BRown, J.L.: Sampling expansions for multiband signals. IEEE Trans. Acoustics, Speech' and Signal Processing 33 (1985), 312 - 315.

[4] BUTZER, P.L.: A survey of the Whittaker-Shannon sampling theorem and some of its extensions. J. Math. Res. Exp. 3 (1983), 185 - 212.

[5] Butzer, P.L., Splettstoesser, W. and R.L. Stens: The Sampling Theorem and Linear Prediction in Signal Analysis. Jahresber. Dt. Math.-Verein. 90 (1988), 1 - 70.

[6] Dodson, M.M., and A.M. SILVA: An algorithm for optimal sampling rates for multiband signals. Signal Processing 17 (1989), $169-174$.

[7] Dodson, M.M.:Diophantine inequalities and sampling rates for multiband signals. In: Recent Advances in Fourier Analysis and its Applications (eds.: J.S. Byrnes and J.F. Byrnes). Dordrecht-Boston-London: Kluwer Academic Publishers 1990, pp. 483 - 498.

[8] DYM, H., and H.P. MCKEAN: Fourier Series and Integrals. New York and London: Academic Press 1972.

[9] Garnett, J.B.: Bounded Analytic Functions. New York and London: Academic Press 1981.

[10] Gohberg, I.Ts., and M.G. KREIN: Introduction to the Theory of Linear Nonselfadjoint Operators in Hilbert Space (in Russian). Moscow: Nauka 1965. Engl. transl.: Providence, R.I.: Amer. Math. Soc. 1969.

[11] Goldman, S.: Information Theory. New York: Prentice-Hall 1953.

[12] HARDY, G.H.: Notes on special systems of othogonal functions, IV: The orthogonal functions of Whittaker's cardinal series. Proc. Camb. Phil. Soc. 37 (1941), 331 - 348. Reprinted in: Collected papers of G.H.Hardy, Vol.3. Oxford: The Clarendon Press 1969, pp. $466-483$.

[13] Higgins, J.R.: Five short stories about the cardinal series. Bull. Amer. Math. Soc. $12(1985), 45-89$.

[14] Higgins, J.R.: Some gap sampling series for multiband signals. Signal Processing 12 (1987), $313-319$.

[15] Higgins, J.R.: Sampling theory for Paley - Wiener spaces in the Riesz basis setting. Proc. Royal Irish. Acad. (to appear).

[16] HRUŚCEV, S.V., Nikolskil, N.K., and B.S. PAVlov: Unconditional bases of exponentials and of reproducing kernels. Lect. Notes Math. 864 (1981), 214 - 335.

[17] JERRI, A.J.: The Shannon sampling theorem - Its various extensions and applications: A tutorial review. Proc. IEEE 65 (1977), 1565 - 1596.

[18] Katsnelson, V.E.: Exponential bases in $L^{2}$ (in Russian). Funktional'nyi analiz i ego prilożeniya 5 (1971)1, 37 - 47. Engl. transl. in: Funct. Anal. Appl. 5 (1971), 31 - 38. 
[19] Khurgin, Ya.I., and V.P. Yakovlev: Finite Functions in Physics and Engineering (in Russian). Moscow: Nauka 1971.

[20] Kotelnikov, V.A.: On the code capacity of 'ether' and wire in the electrical communication (in Russian). In: Vsesojuznii energetičeskii komitet. Materiali k I Vsesojuznomu sjezdu po voprosam tehničeskoi rekonstrukcii dela svjazi i razvitija slabotočnoi promišlennosti. Moscow: Izd. upravlenija svjazi RKK 1933.

[21] LANDAU, H.J.: A sparse regular sequence of exponentials closed on large sets. Bull. Amer. Math. Soc. 70 (1964), 566 - 569.

[22] LANDAU, H.J.: Necessary density conditions for sampling and interpolation of certain entire functions. Acta Math. 117 (1967), 37 - 52.

[23] Levin, B.Ya.: Exponential bases in $L^{2}$ (in Russian). Zap. Mat. Otd. Fiz.-Mat. Fak. Har'k. Un-ta. i Har'k. Mat. Obšč. 27 (1961), 39 - 48.

[24] LEvin, B.Ya.: Interpolation by entire functions of exponential type (in Russian). Mat. Fizika i Funkcional. Analiz 1 (ed.: V.A. Marchenko). Kiev: Naukova Dumka 1972, pp. $136-146$.

[25] NIKOL'SKIl, N.K.: Treatise on the Shift Operator. Berlin-Heidelberg-New York: SpringerVerlag 1986.

[26] NYQUIST, H.: Certain topics in telegraph transmission theory; Trans. Amer. Inst. Electr. Eng. 47 (1928), 617 - 644.

[27] Plancherel, M., and G. Polya: Fonction entières et intégrales de Fourier multiples (Seconde partie) . Comment. Math. Helvetici. 10 (1938), 110 - 163. Reprinted in: G. Pólya: Collected papers, Vol. 1. Cambrige MA and London: The MIT Press 1973, pp. $644-697$

[28] Shannon, C.: Communication in the presence of noise. Proc. IRE 37 (1949), 10 - 21.

[29] WALTER, G.G.: Recent extensions of the sampling theorem. In: Signal processing, Part I: Signal Processing Theory (eds:: L. Auslander, T. Kailath and S. Mitter). BerlinHeidelberg-New York: Springer-Verlag 1990, pp. $229-238$.

[30] WHITTAKER, E.T.: On the functions, which are represented by expansions of the interpolation theory. Proc. Roy. Soc. Edinburgh 35 (1915), 181 - 194.

[31] WHitTAKER, J.M.: Interpolatory Function Theory (Cambridge Tracts in Mathematics and Math. Physics: Vol. 33). Cambridge: Cambridge Univ. Press 1935.

[32] Young, R.M.: An Introduction to Nonharmonic Fourier Series. New York and London: Academic Press 1980. 